\title{
Novel Design and Fabrication of High Sensitivity MEMS Capacitive Sensor Array for Fingerprint Imaging
}

\author{
Mitra Damghanian ${ }^{1, a}$, Burhanuddin Yeop Majlis ${ }^{1, b}$
}

${ }^{1}$ Institute of Microengineering and Nanoelectronics (IMEN), Universiti Kebangsaan Malaysia, 43600, Bangi, Selangor, Malaysia

amitra@eng.ukm.my, ${ }^{\mathrm{b}}$ burhan@eng.ukm.my

Keywords: MEMS capacitive sensors, fingerprint imaging, sensor's sensitivity, sensor array.

Abstract. A novel MEMS capacitive pressure sensor array is designed and fabricated for fingerprint acquisition application. Based on analytical investigations and FEM analysis, the designed structure of pressure sensor cells assist from an aluminum clamped-clamped wide beam as the movable electrode of variant capacitor, instead of usual membrane structure. A rectangular base T-shape protrusion is also used on top of the deflecting electrode to concentrate pressure and increase the sensitivity. Proven by the real test of the fabricated sensor structure, this design has enhanced sensitivity and linearity of the device compared to all membrane based reported prototypes without crossing the dpi limits. Structural modifications have resulted in a simpler fabrication process as well.

\section{Introduction}

Biometrics is a science of automatically identifying individuals based on their unique physiological or behavioral characteristics. Among many different biometric techniques, fingerprint is the most established, studied, widely used and accepted one [1]. Fingerprint sensors are easy to use and at the present time the most common biometric sensors. MEMS devices have been the recent interest in fingerprint acquisition systems. They are going to have an outstanding market share among fingerprint imagers according to their significant characteristics such as their low power consumption, durability, being resistant to electro static discharge (ESD) and more over, their non bulky nature. Some recent works are listed in [1]. Nowadays MEMS products have a market share of over 20 billion USD a year [2]. Currently, fingerprint sensors are widely used in cell phones and PCs and are looking forward to an enormous market opportunity by being integrated into credit cards, smart cards and other identity and security cards. Capacitive MEMS fingerprint imagers offer many advantages over other existing technologies such as optical or ultrasonic imagers, which consequently makes them a promising alternative in the future market where low cost, highly sensitive and thin fingerprint imagers are required. Hereby, the design and fabrication of a high sensitivity MEMS capacitive sensor array for fingerprint imaging is presented followed by the measurements on sensitivity and linearity of the fabricated structure.

\section{Designed Structure}

Basic Equations. The following equations explain the basic behavior of the capacitive pressure sensor cells along with the corresponding sensitivity $(S)$ and linearity $(L)$ definitions:

$$
\begin{aligned}
& \Delta C=C_{f}-C_{i}=\varepsilon\left(\int_{0}^{b} \int_{0}^{a} \frac{1}{g-w(x, y)} d x d y-\frac{a b}{g}\right), \\
& S_{w}=\frac{\partial C}{\partial P}, \quad S_{\text {ave }}=\Delta C_{\text {active range }} / \Delta P_{\text {active range }}
\end{aligned}
$$




$$
L=100-\frac{\left|\Delta C_{+}\right|+\left|\Delta C_{-}\right|}{2\left(C_{\text {max }}-C_{\text {min }}\right)} \times 100 .
$$

Pressure applied on the movable electrode $(P)$ is converted to the mechanical deflection of this suspended thin structure $(w)$, which makes a proportional capacitance change $\left(C_{f}-C_{i}\right)$ read out by the circuit module. $\Delta C_{+}$and $\Delta C_{-}$indicate the maximum deviations of the capacitance from the least square line. A graph representing the induced capacitance change versus the applied pressure in the active pressure range of the device is known as the behavioral characteristic of the device. Definition of parameters can be found in Fig. 1 and Fig. 2.

Figure 1 A typical C-P characteristic graph of a capacitive pressure sensor in normal region

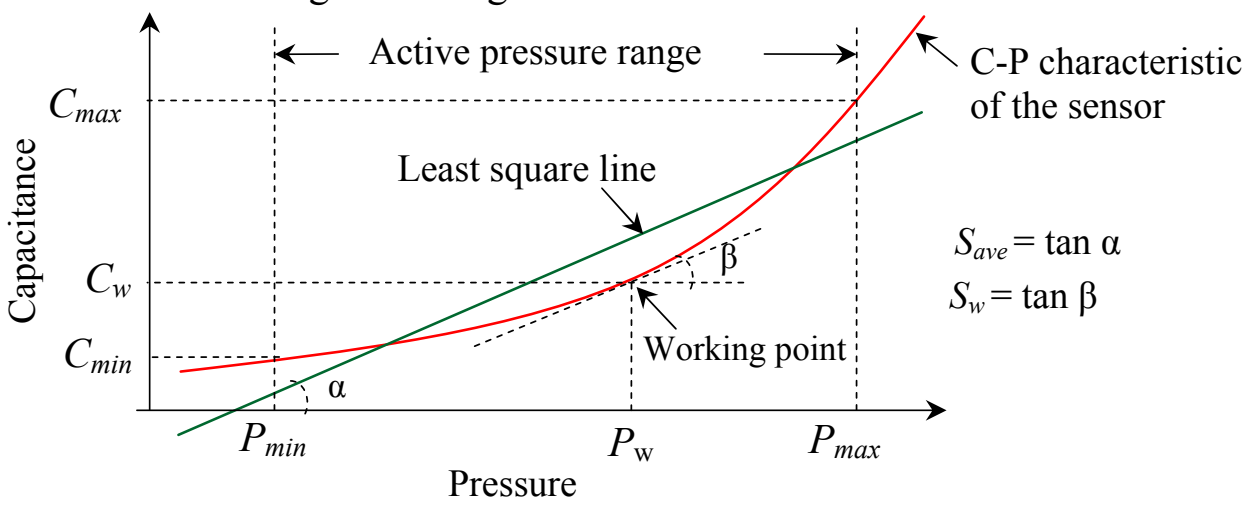

Structure of One Capacitive Cell. A wide beam structure is utilized as the deflecting electrode of each capacitive cell. Deflection properties of this suspended structure based on [3] is the key feature of the superior behavior of this novel design. To improve the deflecting properties of the wide beam, a rectangular base T-shape protrusion is placed on top of this suspended structure. The protrusion concentrates the applied pressure on the center line of the wide beam as shown in Fig. 2 .

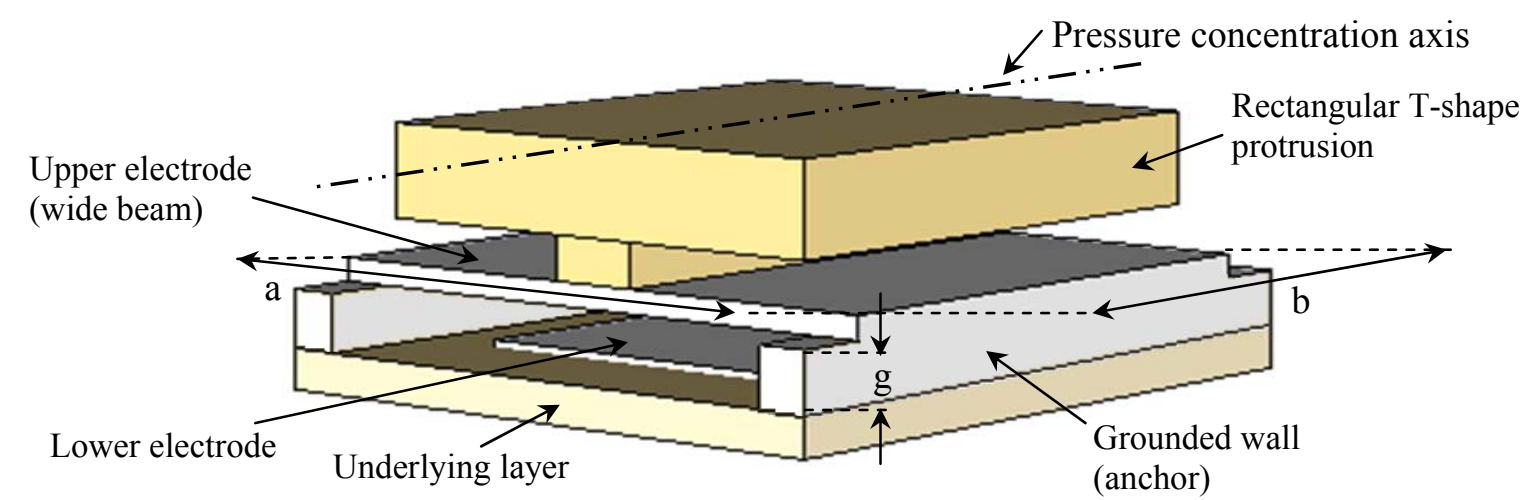

Figure 2 Schematic structure of an individual capacitive cell

Array Concept. For a fingerprint imager it is necessary to have sufficient resolution. Considering each capacitive cell as a pixel in the output fingerprint image, a 500 dpi sensor array is required to satisfy the image constraints, which requires a size of $50 \mu \mathrm{m} \times 50 \mu \mathrm{m}$ for each sensor cell (see Fig. 3).

Applied pressure

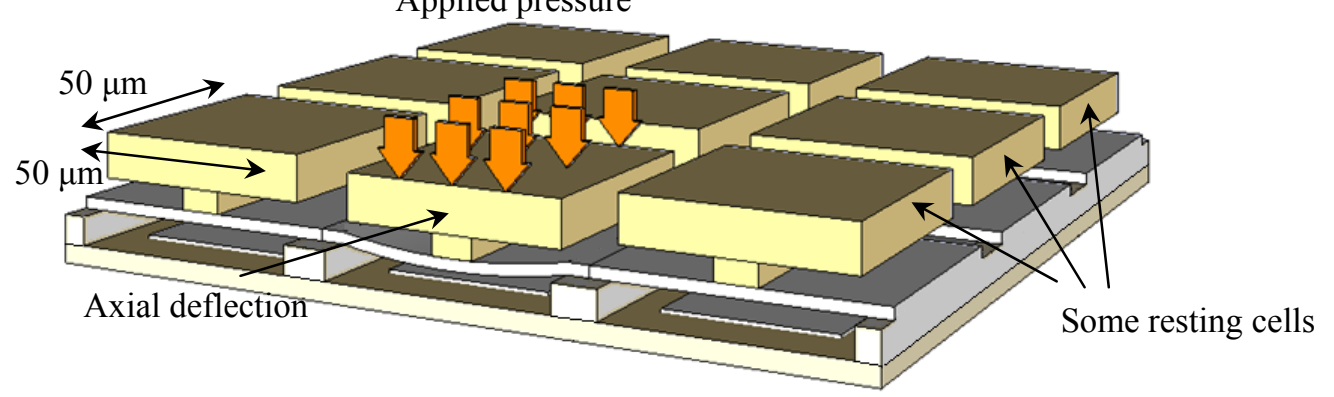

Figure 3 Two dimensional array concept of the fingerprint imager 


\section{Fabrication}

Material Selection. The device is fabricated on glass substrate, using aluminum for lower electrode, signal lines, grounded walls and upper electrodes. A photo-definable thick polyimide is selected for realization of the pressure-concentrating unit on top of the upper electrodes.

Process Flow. The fabrication process starts with a modified lift off technique [4] to create the lower electrode and the signal lines while generating a final flat surface. In this step, after a wet etching process with controlled depth, aluminum deposition and lift off creates the lower electrodes and the signal lines using one single lithography stage (Fig. 4.a). Using this method, no pattern following effect will damage the uniformity of the suspended structure in the following fabrication steps. The process is then followed by deposition and patterning of the sacrificial layer. Photo resist AZ1500 is used as the sacrificial material with $1 \mu \mathrm{m}$ thickness, which reduces the deposition and patterning procedure to a single lithography step. Anchors and the suspended structure are fabricated in the next stage by deposition of a $2 \mu \mathrm{m}$ aluminum layer. A photo sensitive polyimide (PI2720) is utilized to fabricate the protrusion on top of the upper electrode. Once again, modified lift off technique is utilized for fabrication of the protrusion base area and cap at the same time. In this method, first a thick photo resist is spin coated, baked, exposed and developed to form the free space for the protrusion base area. Subsequently, the protrusion base and cap will be fabricated by spin coating, baking, exposing and developing of the photo definable polyimide (PI2720). The final stage is then to remove the thick resist and the sacrificial layer (AZ1500) using PRS2000 solution in $70^{\circ} \mathrm{C}$. The photo image of a 6 cell fabricated structure is illustrated in Fig. 4.b.

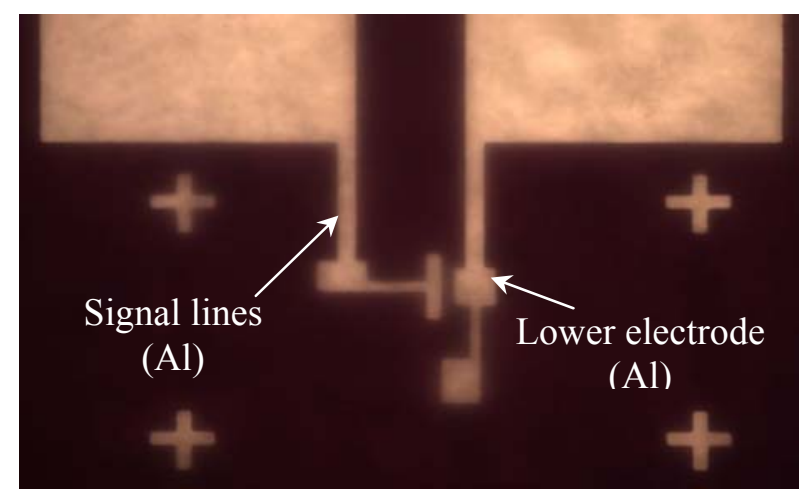

(a)

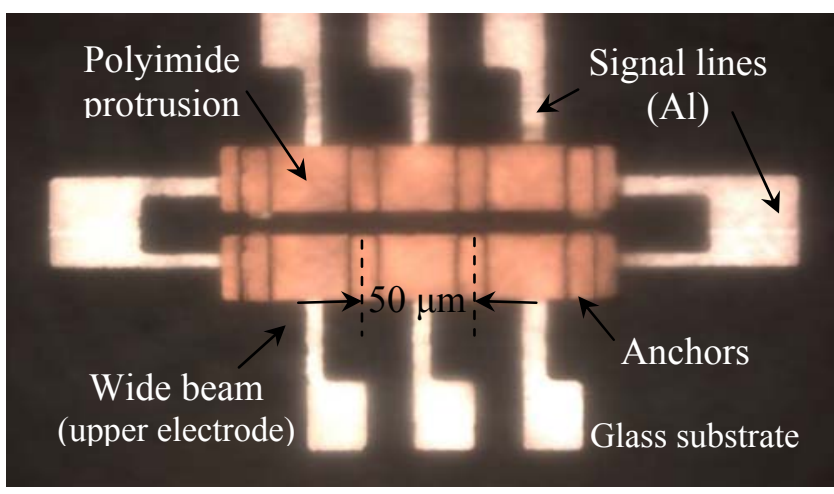

(b)

Figure 4 (a) Photo image of the lower electrode and signal lines of one single cell after the first modified lift off step (b) fabricated array of $2 \times 3$ capacitive cells

\section{Measurements}

Measurements are conducted in two steps, first with the aid of a 4-point probe station for single cell measurements and next by using a 13 channel capacitance to digital converter IC (AD7147), connected to a one dimensional array of 13 cells and a microcontroller board connected to PC, to illustrate the performance of the structure in the array shape (Fig. 5).

First of all, static measurements of the cell capacitance are done with no applied pressure on the cell. The dynamic measurements in the next step are simplified to discrete points where weight loads of the proper amounts are put on one sensing cell structure. The weight loads are calculated to simulate the exact pressure on the protrusion with respect to the protrusion cap area.

Fig. 6 shows a comparison between the FEM simulation results and the results obtained from measurements. Average sensitivity of the device and its linearity are extracted from the measured C-P characteristics based on Eq. 2 and 3. The obtained sensitivity and linearity based on a $1 \mu \mathrm{m}$ gap size and $2 \mu \mathrm{m}$ thickness of the upper electrode are equal to $36 \mathrm{fF} / \mathrm{MPa}$ and $91 \%$, while calculated sensitivity using a membrane with the same geometrical properties is only $7 \mathrm{fF} / \mathrm{MPa}$ (see Fig. 6). 


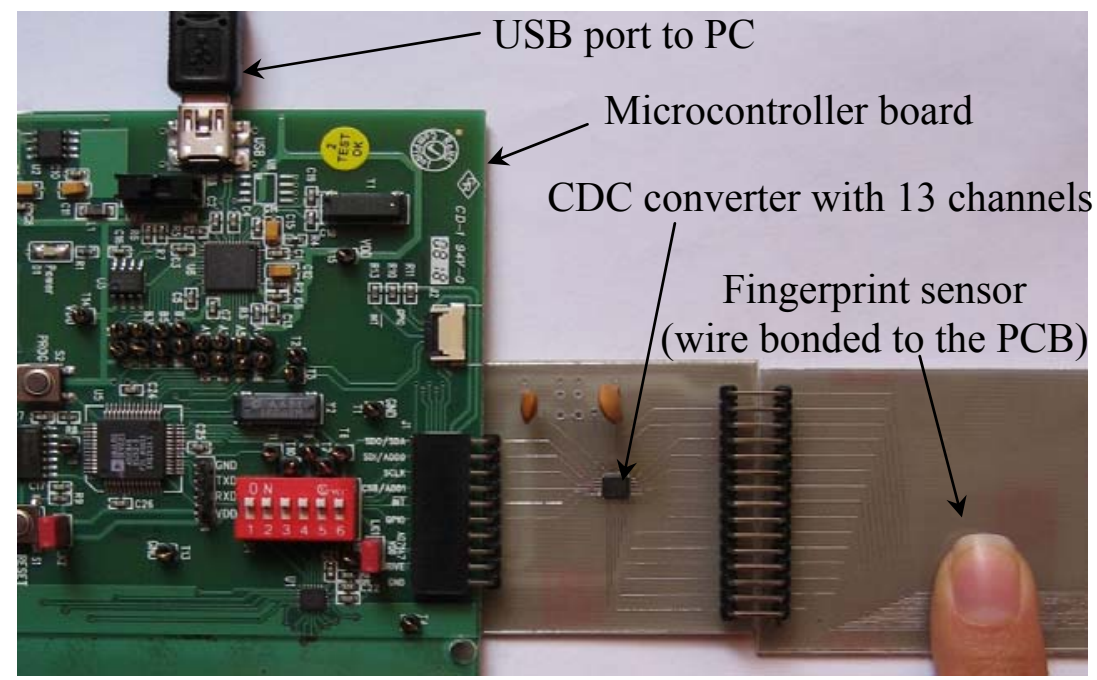

Figure 5 System setup for dynamic measurements

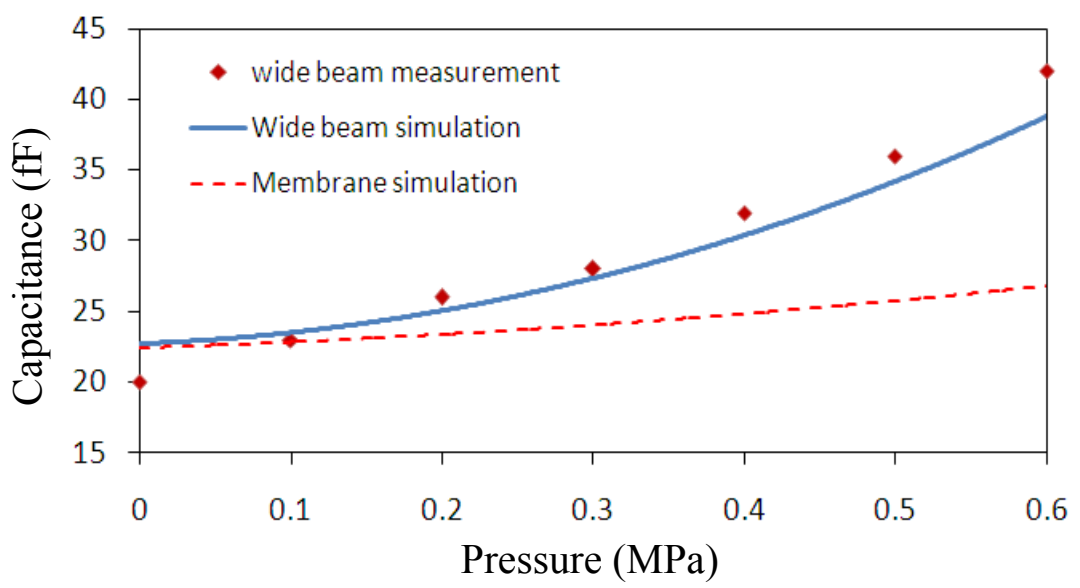

Figure 6 Comparrison between simulations and measurement results

\section{Summary}

An array of MEMS capacitive sensor is designed and fabricated particularly for fingerprint imaging application. The highly sensitive design stage assisting from a wide beam structure was followed by a low cost / low temperature fabrication process to fulfill the device requirements as a promising alternative in the future market of thin fingerprint sensors. The measurements on the fabricated sensor array show a good agreement with results from the simulations. A measured sensitivity of 36 $\mathrm{fF} / \mathrm{MPa}$ and linearity of $91 \%$ was obtained with the sensor cells having $1 \mu \mathrm{m}$ gap size and $2 \mu \mathrm{m}$ beam thickness. The structure has shown superior potential in the applications requiring thin structure and high sensitivity and linearity at the same time.

\section{References}

[1] Nanavati, S., Thieme, M., Nanavati, R. in: Biometrics: Identity Verification in a Networked World. New York, John Wiley \& Sons Inc., ISBN 0471-09945-7 (2002).

[2] NEXUS, “The NEXUS MST/MEMS Market Analysis III 2005 - 2009,” Neuchâtel: European Microsystems Network, (2005).

[3] M.damghanian, B. Y. Majlis: Analysis and design of a wide micro beam as a pressure gauge for high sensitivity MEMS fingerprint sensors. Journal of Microsystem Technology (2009), in press.

[4] M.damghanian, B. Y. Majlis: A Modified Lift-off Technique to Prevent Pattern Following Effect in Microfabrication. ICSE (2008) Proc., Johor Bahru, Malaysia. 